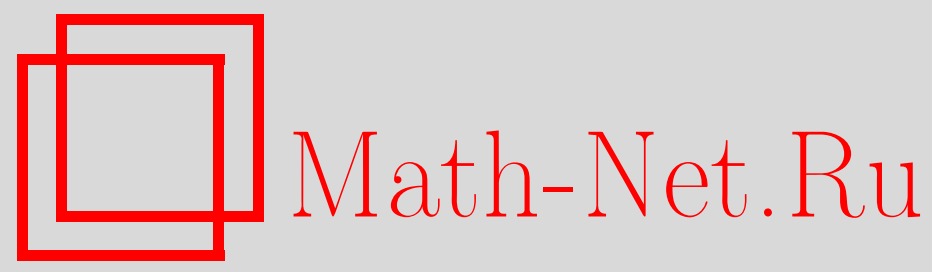

А. А. Серов, Оценка числа булевых функций, имеющих квадратичные приближения заданной точности, Дискрет. матем., 2012, том 24, выпуск 3, 90-107

DOI: https://doi.org/10.4213/dm1201

Использование Общероссийского математического портала Math-Net.Ru подразумевает, что вы прочитали и согласны с пользовательским соглашением http://www . mathnet.ru/rus/agreement

Параметры загрузки:

IP : 54.196 .121 .252

26 апреля 2023 г., 12:20:09 
УДК 519.7

\title{
Оценка числа булевых функций, имеющих квадратичные приближения заданной точности
}

() 2012 г. A. A. Серов

\begin{abstract}
Получены двусторонние оценки и асимптотические формулы для числа булевых функций от $n$ переменных, которые с заданной точностью аппроксимируются квадратичными булевыми функциями.

Работа выполнена при поддержке Российского фонда фундаментальных исследований, проект 11-01-00139.
\end{abstract}

\section{1. Введение}

Пусть $\mathbb{F}_{2}$ - поле из двух элементов. Для произвольного натурального числа $n$ будем обозначать через $V_{n}=\mathbb{F}_{2}^{n}$ пространство $n$-мерных векторов с компонентами из $\mathbb{F}_{2}$. Между множеством

$$
\mathbb{F}_{2}^{V_{n}}=\left\{f: V_{n} \rightarrow \mathbb{F}_{2}\right\}
$$

всех булевых функций $n$ переменных и пространством $V_{2^{n}}$ можно установить взаимно однозначное соответствие, отождествляя функцию $f \in \mathbb{F}_{2}^{V_{n}}$ с вектором $\left\{f(x) \mid x \in V_{n}\right\}$.

Расстояние Хэмминга $\rho(f, g)$ между булевыми функциями $f, g \in \mathbb{F}_{2}^{V_{n}}$ определяется как число значений аргумента $x \in V_{n}$, при которых $f(x) \neq g(x)$. Для произвольного множества булевых функций $A \subset \mathbb{F}_{2}^{V_{n}}$ и функции $f \in \mathbb{F}_{2}^{V_{n}}$ обозначим через

$$
\rho(f, A)=\min _{g \in A} \rho(f, g)
$$

расстояние Хэмминга от $f$ до ближайшей к ней функции из множества $A$.

В множестве $\mathbb{F}_{2}^{V_{n}}$ всех булевых функций выделяется класс квадратичных функций

$$
\mathbb{Q}_{n}=\left\{f \in \mathbb{F}_{2}^{V_{n}}: f\left(x_{1}, \ldots, x_{n}\right)=\bigoplus_{1 \leqslant i<j \leqslant n} b_{i j} x_{i} x_{j} \oplus \bigoplus_{i=1}^{n} a_{i} x_{i} \oplus a_{0}, b_{i j}, a_{i} \in \mathbb{F}_{2}\right\},
$$

где $\oplus-$ сложение в $\mathbb{F}_{2}$. Множество $\mathbb{Q}_{n}$ состоит из $\left|\mathbb{Q}_{n}\right|=2^{\left(\begin{array}{c}n \\ 2\end{array}\right)+n+1}$ элементов:

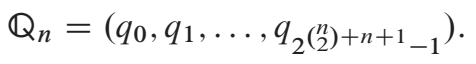


Далее для упрощения записи формул будем использовать обозначения:

$$
n_{s}^{-}=2^{n-2}-2^{n-s-2}, \quad n_{s}^{+}=2^{n-2}+2^{n-s-2},
$$

а также обозначение $W_{i}$ для множества функций веса $i$ из $\mathbb{Q}_{n}$, то есть

$$
W_{i}=\left\{f \in \mathbb{Q}_{n}: \rho(f, 0)=i\right\} .
$$

Известно (см. [3], с. 426), что

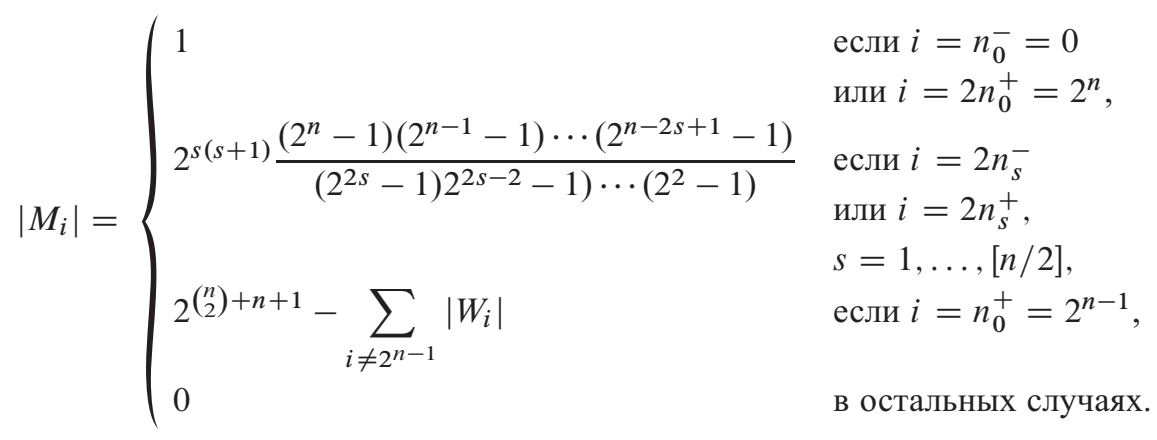

Замечание 1. Справедливы оценки

$$
\begin{aligned}
\left|W_{2 n_{s}^{-}}\right| & <3 \cdot 2^{2 n s-2 s^{2}+s-1}, \quad s=1, \ldots,[n / 2]-1, \\
\left|W_{2 n_{[n / 2]}^{-}}\right| & <2^{2 n[n / 2]-2[n / 2]^{2}+[n / 2]} \\
& = \begin{cases}2^{2 k^{2}+k} & \text { если } n=2 k ; \\
2^{2 k^{2}+3 k} & \text { если } n=2 k+1 .\end{cases}
\end{aligned}
$$

Доказательство. Нетрудно показать, что

$$
\begin{aligned}
\prod_{i=1}^{s} \frac{\left(2^{n-2 s+2 i}-1\right)\left(2^{n-2 s+2 i-1}-1\right)}{2^{2 i}-1} & <2^{2 n s-4 s^{2}} \prod_{i=1}^{s} \frac{2^{4 i-1}}{2^{2 i}-1} \\
& =2^{2 n s-4 s^{2}} \prod_{i=1}^{s} \frac{2^{2 i-1}}{1-1 / 2^{2 i}} \\
& =2^{2 n s-3 s^{2}} \prod_{i=1}^{s} \frac{1}{1-1 / 2^{2 i}}
\end{aligned}
$$

и

$$
\prod_{i=1}^{s}\left(1-\frac{1}{2^{2 i}}\right) \geqslant 1-\sum_{i=1}^{s} \frac{1}{2^{2 i}}=1-\frac{2}{3}+\frac{1}{3 \cdot 4^{s}}
$$

Тогда

$$
\begin{aligned}
\left|W_{2 n_{s}-}\right| & =2^{s(s+1)} \prod_{i=1}^{s} \frac{\left(2^{n-2 s+2 i}-1\right)\left(2^{n-2 s+2 i-1}-1\right)}{2^{2 i}-1} \\
& <2^{2 n s-2 s^{2}+s} \frac{3}{2+1 / 4^{s}} .
\end{aligned}
$$


Теперь докажем, что при $s=[n / 2]$ для сомножителей в левой части (3) имеет место неравенство

$$
\frac{\left(2^{n-2 s+2 i}-1\right)\left(2^{n-2 s+2 i-1}-1\right)}{2^{2 i}-1}<\frac{2^{n-2 s+2 i} 2^{n-2 s+2 i-1}}{2^{2 i}} .
$$

Неравенства, эквивалентные (4), имеют вид

$$
\begin{aligned}
2^{2 n-4 s+4 i-1}-2^{n-2 s+2 i}-2^{n-2 s+2 i-1}+1<2^{2 n-4 s+2 i-1}\left(2^{2 i}-1\right), \\
2^{2 n-4 s+2 i-1}-3 \cdot 2^{n-2 s+2 i-1}+1<0 .
\end{aligned}
$$

Неравенство (5) при четном $n$ эквивалентно неравенству

$$
1-3+1 / 2^{2 i-1}<0
$$

а при нечетном $n$ эквивалентно неравенству

$$
4-3 \cdot 2+1 / 2^{2 i-1}<0
$$

так как $1 / 2^{2 i-1}<2$ для любого $i \in\{1, \ldots, s\}$, что доказывает (4).

Значит, при $s=[n / 2]$

$$
\begin{aligned}
\left|W_{2 n_{s}}\right| & <2^{s(s+1)} \prod_{i=1}^{s} \frac{2^{n-2 s+2 i} 2^{n-2 s+2 i-1}}{2^{2 i}}=2^{s(s+1)} \prod_{i=1}^{s} 2^{2 n-4 s+2 i-1} \\
& =2^{s(s+1)} 2^{2 s n-4 s^{2}+s(s+1)-s}=2^{2 s n-2 s^{2}+s} \\
& = \begin{cases}2^{2 k^{2}+k} & \text { если } n=2 k, \\
2^{2 k^{2}+3 k} & \text { если } n=2 k+1 .\end{cases}
\end{aligned}
$$

Замечание 1 доказано.

Пусть

$$
D_{n}=\left\{2^{n-1}\right\} \cup\left\{2 n_{s}^{ \pm}, s=0,1, \ldots,[n / 2]\right\}
$$

- множество всех $i$ таких, что $\left|W_{i}\right| \neq 0$, тогда

$$
\mathbb{Q}_{n}=W_{2^{n-1}} \bigcup_{i \in D_{n}} W_{i} .
$$

Пусть

$$
\mathbb{F}_{2}\left(\mathbb{Q}_{n}, r\right)=\left\{f \in \mathbb{F}_{2}^{V_{n}}: \rho\left(f, \mathbb{Q}_{n}\right) \leqslant r\right\}
$$

- множество всех булевых функций от $n$ переменных, расстояние от которых до множества $\mathbb{Q}_{n}$ квадратичных функций меньше или равно $r$.

В [4] доказаны предельные теоремы для распределения расстояния от случайной булевой функции до множества $\mathbb{Q}_{n}$. В частности, в [4] показано, что для любого фиксированного $x$

$$
\lim _{n \rightarrow \infty} \mathbf{P}\left\{\frac{\rho\left(f, \mathbb{Q}_{n}\right)-a_{n}}{b_{n}} \leqslant x\right\}=1-e^{-e^{x}},
$$


где

$$
\begin{aligned}
& a_{n}=2^{n-1}-2^{(n-2) / 2} n \sqrt{\ln 2}\left\{1+\frac{1}{2 n}-\frac{4 \ln \left(\pi n^{2} \ln 2\right)-\ln 2}{8 n^{2} \ln 2}\right\}, \\
& b_{n}=\frac{2^{(n-2) / 2}}{n \sqrt{\ln 2}} .
\end{aligned}
$$

Из(6) и (7) следует, что если $\mathbb{F}_{2}\left(\mathbb{Q}_{n}, r\right)$ - множество всех булевых функций, расстояние Хэмминга от которых до множества квадратичных функций не превосходит $r$, то при $n \rightarrow \infty$

$$
\sup _{0 \leqslant r<2^{n-1}-2^{n / 2-1} \sqrt{\ln 2}}\left|2^{-2^{n}}\right| \mathbb{F}_{2}\left(\mathbb{Q}_{n}, r\right)\left|-\left(1-e^{-e^{-x(n, r)}}\right)\right| \rightarrow 0,
$$

где $x(n, r)$ определяется равенством

$$
r=2^{n-1}-2^{n / 2-1} \sqrt{\left(n^{2} \ln 2+n \ln 2-2 \ln n-\ln (\pi \ln 2)+\ln \sqrt[4]{2}+2 x(n, r)\right)} ;
$$

Иными словами, для основной массы булевых функций от $n$ переменных расстояние до множества квадратичных функций имеет вид $2^{n-1}-\sqrt{2^{n-2}\left(n^{2} \ln 2+n \ln 2-2 \ln n\right)}+$ $O\left(2^{n / 2} / n\right)$ при $n \rightarrow \infty$.

В настоящей работе методом включения-исключения получены точные формулы и верхние и нижние оценки для числа элементов множества $\mathbb{F}_{2}\left(\mathbb{Q}_{n}, r\right)$. Один из основных результатов (объединение части формулировок теорем 2, 3 и предложения 2) можно сформулировать следующим образом.

Теорема 1. Если $n \geqslant 3$, то

$$
(1-Q(n, r)) 2^{\left(\begin{array}{c}
n \\
2
\end{array}\right)+n+1} \sum_{m=0}^{r}\left(\begin{array}{l}
2^{n} \\
m
\end{array}\right) \leqslant\left|\mathbb{F}_{2}\left(\mathbb{Q}_{n}, r\right)\right| \leqslant 2^{\left(\begin{array}{c}
n \\
2
\end{array}\right)+n+1} \sum_{m=0}^{r}\left(\begin{array}{l}
2^{n} \\
m
\end{array}\right),
$$

где $Q(n, r)=0$ при $0 \leqslant r<2^{n-3}$,

$$
Q(n, r)<\frac{2^{-n^{2}\left(c_{r}^{2}-3\right) / 6+n+1}}{n^{2}} \exp \left\{\frac{\left.c_{r} n\right)^{3}}{7 \cdot 2^{n / 2}}\right\}
$$

при $n \geqslant 15, u$

$$
r=2^{n-1}-c_{r} n \sqrt{2^{n-2} \ln 2} \geqslant 0, \quad c_{r}>1 .
$$

Замечание 2. Теорема 1 позволяет получать оценки левого хвоста распределения расстояния от случайной булевой функции до множества квадратичных булевых функций.

Отношения верхних и нижних оценок в теореме 1 стремятся к 1 , если $n \rightarrow \infty$ и

$$
r<2^{n-1}-c n \sqrt{2^{n-2} \ln 2}, \quad c>\sqrt{3}=1,7321 \ldots .
$$

Для сумм $\sum_{m=0}^{r}\left(\begin{array}{c}2^{n} \\ m\end{array}\right)$ при $r \leqslant 2^{n-1}$ в [1] (см. §2) получены явные верхние и нижние оценки, и с их помощью показано, что в области больших уклонений, где $1-e^{-e^{-x(n, r)}} \rightarrow 0$, относительная погрешность приближения величины $2^{-2^{n}}\left|\mathbb{F}_{2}\left(\mathbb{Q}_{n}, r\right)\right|$ величиной $1-e^{-e^{-x(n, r)}}$ быстро растет с уменьшением $r$. 
Следствие 1. Если с > 1 u $n, r \rightarrow \infty$ так, что выполняется условие

$$
r<2^{n-1}-c \sqrt{2^{n-2}\left(n^{2}+n\right) \ln 2},
$$

то при достаточно больщих $n$

$$
\frac{\left|\mathbb{F}_{2}\left(\mathbb{Q}_{n}, r\right)\right|}{2^{2^{n}}\left(1-e^{-e^{-x(n, r)}}\right)}<\frac{3}{2 c}
$$

\section{2. Неравенства включения-исключения}

Считая равными нулю суммы, в которых верхний предел суммирования меньше нижнего, введем

$$
\begin{aligned}
N_{2^{n}}^{(1)}(r) & =\sum_{m=0}^{r}\left(\begin{array}{c}
2^{n} \\
m
\end{array}\right), \\
N_{2^{n}}^{(2)}(k, r) & =\left|\left\{f \in \mathbb{F}_{2}^{V_{n}}: \rho(f, 0) \leqslant r, \rho(f, g) \leqslant r, \rho(g, 0)=k\right\}\right|, \\
A_{n}^{-}(t, r) & =2^{\left(\begin{array}{c}
n \\
2
\end{array}\right)+n} \sum_{s=1}^{t}\left|W_{2 n_{s}^{-}}\right| N_{2^{n}}^{(2)}\left(2 n_{s}^{-}, r\right), \\
A_{n}^{+}(t, r) & =2^{\left(\begin{array}{c}
n \\
2
\end{array}\right)+n} \sum_{s=t}^{[n / 2]}\left|W_{2 n_{s}^{+}}\right| N_{2^{n}}^{(2)}\left(2 n_{s}^{+}, r\right), \\
B_{n}(r) & =2^{\left(\begin{array}{c}
n \\
2
\end{array}\right)+n+1}\left(2^{\left(\begin{array}{c}
n \\
2
\end{array}\right)+n}-\sum_{s=1}^{[n / 2]}\left|W_{2 n_{s}^{-}}\right|-1\right) N_{2^{n}}^{(2)}\left(n_{0}^{+}, r\right),
\end{aligned}
$$

и

$$
S_{n}^{(2)}(r)=\sum_{\substack{q_{1}, q_{2} \in \mathbb{Q}_{n} \\ q_{1} \neq q_{2}}}\left|\left\{f \in \mathbb{F}_{2}^{V_{n}}:: \rho\left(f, q_{1}\right) \leqslant r, \rho\left(f, q_{2}\right) \leqslant r\right\}\right| .
$$

Теорема 2. (a) Если $0 \leqslant r<2^{n-1}$, то

$$
2^{\left(\begin{array}{c}
n \\
2
\end{array}\right)+n+1} N_{2^{n}}^{(1)}(r)-S_{n}^{(2)}(r) \leqslant\left|\mathbb{F}_{2}\left(\mathbb{Q}_{n}, r\right)\right| \leqslant 2^{\left(\begin{array}{c}
n \\
2
\end{array}\right)+n+1} N_{2^{n}}^{(1)}(r),
$$

где

$$
S_{n}^{(2)}(r)= \begin{cases}0 & \text { если } r<n_{1}^{-}, \\ A_{n}^{-}(t, r) & \text { если } n_{t}^{-} \leqslant r<n_{t+1}^{-} \\ A_{n}^{-}([n / 2], r) & \text { и } t=1, \ldots,[n / 2]-1, \\ A_{n}^{-}([n / 2], r)+B_{n}(r) & \text { если } n_{[n / 2]}^{-} \leqslant r<2^{n-2}, \\ A_{n}^{-}([n / 2], r)+B_{n}(r)+A_{n}^{+}(t, r) & \text { если } 2^{n-2} \leqslant r<n_{[n / 2]}^{+}, \\ A_{n}^{-}([n / 2], r)+B_{n}(r)+A_{n}^{+}(1, r) & \text { если } n_{t}^{+} \leqslant r n_{t-1}^{+} \\ \text {и } t=2, \ldots,[n / 2], & \text { если } n_{1}^{+} \leqslant r<2^{n-1} .\end{cases}
$$


(б) Если $0 \leqslant r<2^{n-3}$, то

$$
\left|\mathbb{F}_{2}\left(\mathbb{Q}_{n}, r\right)\right|=2^{\left(\begin{array}{c}
n \\
2
\end{array}\right)+n+1} N_{2^{n}}^{(1)}(r) .
$$

Доказательство. Так как

$$
\mathbb{F}_{2}\left(\mathbb{Q}_{n}, r\right)=\bigcup_{j=0}^{2\left(\begin{array}{c}
n \\
2
\end{array}\right)+n+1}-1
$$

то, согласно формуле включения-исключения (см. [5]),

$$
\left|\mathbb{F}_{2}\left(\mathbb{Q}_{n}, r\right)\right|=\sum_{k=1}^{2\left(\begin{array}{c}
n \\
2
\end{array}\right)+n+1}(-1)^{k-1} \sum_{0 \leqslant i_{1}<\ldots<i_{k}<2}\left|\left\{f \in \mathbb{F}_{2}^{V_{n}}: \max _{1 \leqslant j \leqslant k} \rho\left(f, q_{i_{j}}\right) \leqslant r\right\}\right|,
$$

а значения неполных сумм в (12) по $k$ от 1 до $m \in\left\{1,2, \ldots, 2^{\left(\begin{array}{c}n \\ 2\end{array}\right)+n+1}\right\}$ дают верхние (при нечетных $m$ ) и нижние (при четных $m$ ) оценки для $\left|\mathbb{F}_{2}\left(\mathbb{Q}_{n}, r\right)\right|$. Поэтому для доказательства теоремы достаточно получить формулы для чисел и значений слагаемых в правой части (12), соответствующих $k=1,2$.

При $k=1$ слагаемые в (12) имеют вид

$$
\left|\left\{f \in \mathbb{F}_{2}^{V_{n}}: \rho\left(f, q_{j}\right) \leqslant r\right\}\right|=\sum_{m=0}^{r}\left(\begin{array}{l}
2^{n} \\
m
\end{array}\right)=N_{2^{n}}^{(1)}(r) .
$$

Число таких слагаемых в (12) равно $2^{\left(\begin{array}{c}n \\ 2\end{array}\right)+n+1}$. Поэтому

$$
\begin{aligned}
\left|\mathbb{F}_{2}\left(\mathbb{Q}_{n}, r\right)\right| & \leqslant \sum_{j=0}^{2\left(\begin{array}{c}
n \\
2
\end{array}\right)+n+1}-1 \\
\sum_{m=0} & \left|\left\{f \in \mathbb{F}_{2}^{V_{n}}: \rho\left(f, q_{j}\right) \leqslant r\right\}\right| \\
& =2^{\left(\begin{array}{c}
n \\
2
\end{array}\right)+n+1} \sum_{m}^{r}\left(\begin{array}{c}
2^{n} \\
m
\end{array}\right)=2^{\left(\begin{array}{c}
n \\
2
\end{array}\right)+n+1} N_{2^{n}}^{(1)}(r) ;
\end{aligned}
$$

тем самым, доказана оценка сверху в части (а) теоремы.

В силу $(1), \rho\left(q_{i}, q_{j}\right) \geqslant 2^{n-2}$ при любых

$$
\rho\left(q_{i}, q_{j}\right) \geqslant 2^{n-2}, \quad 0 \leqslant i<j<2^{\left(\begin{array}{c}
n \\
2
\end{array}\right)+n+1} ;
$$

значит,

$$
\rho\left(f, q_{i}\right)+\rho\left(f, q_{j}\right) \geqslant 2^{n-2}
$$

для любой функции $f \in \mathbb{F}_{2}^{V_{n}}$, то есть,

$$
\max \left\{\rho\left(f, q_{i}\right), \rho\left(f, q_{j}\right)\right\} \geqslant 2^{n-3} .
$$

Поэтому в сумме (12) при $r<2^{n-3}$ все слагаемые с $k>1$ равны 0 , и тем самым доказано утверждение (б).

Если выбор квадратичных функций $q_{i_{1}}, \ldots, q_{i_{k}} \in \mathbb{Q}_{n}$ таков, что $q_{i_{u}}(x)+q_{i_{v}}(x)=1$ для всех $x \in V_{n}$ и некоторого $1 \leqslant u<v \leqslant k$, то для любой булевой функции $f \in \mathbb{F}_{2}^{V_{n}}$ справедливо равенство

$$
\rho\left(f, q_{i_{u}}\right)+\rho\left(f, q_{i_{v}}\right)=2^{n}
$$


следовательно, в этом случае

$$
\max _{1 \leqslant j \leqslant k} \rho\left(f, q_{i_{j}}\right) \geqslant 2^{n-1},
$$

иными словами, при $r<2^{n-1}$

$$
\left|\left\{f \in \mathbb{F}_{2}^{V_{n}}: \max _{1 \leqslant j \leqslant k} \rho\left(f, q_{i_{j}}\right) \leqslant r\right\}\right|=0 .
$$

Поэтому в дальнейшем в сумме (12) рассматриваются только наборы попарно различных квадратичных булевых функций, попарные суммы которых отличны от функции, тождественно равной 1 .

Многочлены Жегалкина функции $q(x) \in \mathbb{Q}_{n}, x=\left(x_{1}, \ldots, x_{n}\right) \in V_{n}$, можно записать в матричном виде

$$
q(x)=x Q_{q} x^{T} \oplus a_{q} x^{T} \oplus a_{0},
$$

где $T$ - оператор транспонирования, $Q_{q}=\left(q_{i j}\right)$ - верхнетреугольная двоичная $(n \times n)$ матрица с нулевой главной диагональю, $a_{q}=\left(a_{1}, a_{2}, \ldots, a_{n}\right), a_{i} \in \mathbb{F}_{2}$. С матрицей $Q_{q}$ связана симметричная матрица

$$
\widetilde{Q}_{q}=Q_{q} \oplus Q_{q}^{T},
$$

называемая симплектической матрицей.

Известно (см., например, [3], с. 422, или [2], с. 146), что если ранг симплектической формы $\widetilde{Q}_{q}$, соответствующей $q$, равен $2 s$, то существует такое линейное невырожденное преобразование $t: V_{n} \rightarrow V_{n}$, что $q^{t}(x)=q(t(x))$ имеет один из перечисленных ниже видов:

(a) если $\operatorname{rank} \widetilde{Q}_{q}=0$, то либо $q^{t}(x)=a_{0}, a_{0} \in \mathbb{F}_{2}$, либо $q^{t}(x)=x_{1}$;

(б) если $\operatorname{rank} \widetilde{Q}_{q}=2 s, s \geqslant 1$, то либо

$$
q^{t}(x)=\sum_{i=1}^{s} x_{2 i-1} x_{2 i} \oplus a_{0}, \quad a_{0} \in \mathbb{F}_{2},
$$

или

$$
q^{t}(x)=\sum_{i=1}^{s} x_{2 i-1} x_{2 i} \oplus x_{2 s+1}
$$

Далее, заметим, что множество $\mathbb{Q}_{n}$ квадратичных функций образует в $\mathbb{F}_{2}^{V_{n}}$ группу по сложению, то есть является линейным кодом, а именно, линейным кодом Рида-Маллера второго порядка. Поэтому

$$
\begin{aligned}
\left|\left\{f \in \mathbb{F}_{2}^{V_{n}}: \max _{1 \leqslant j \leqslant k} \rho\left(f, q_{i_{j}}\right) \leqslant r\right\}\right| & =\left|\left\{f \in \mathbb{F}_{2}^{V_{n}}: \max _{1 \leqslant j \leqslant k} \rho\left(f \oplus q_{i_{1}}, q_{i_{j}} \oplus q_{i_{1}}\right) \leqslant r\right\}\right| \\
& =\left|\left\{f \in \mathbb{F}_{2}^{V_{n}}: \max _{1 \leqslant j \leqslant k} \rho\left(f, q_{i_{j}} \oplus q_{i_{1}}\right) \leqslant r\right\}\right|
\end{aligned}
$$

для любого набора функций $q_{i_{1}}, \ldots, q_{i_{k}} \in \mathbb{Q}_{n}$, то есть при вычислении значений слагаемых в (12) достаточно рассматривать только наборы квадратичных функций, содержащие нулевую функцию, поскольку $q_{i_{1}} \oplus q_{i_{1}}=0$. 
Значит, при $k=2$ слагаемые в (12) есть $\left|\left\{f \in \mathbb{F}_{2}^{V_{n}}: \rho(f, 0) \leqslant r, \rho(f, q) \leqslant r\right\}\right|$.

Если число квадратичных функций веса $y>0$ есть $\left|W_{y}\right|$, то число упорядоченных пар квадратичных функций, находящихся на расстоянии $y$ друг от друга, равно $2^{\left(\begin{array}{c}n \\ 2\end{array}\right)+n+1}\left|W_{y}\right|$, а неупорядоченных пар - в два раза меньше. Если $y=2^{n}$, то сумма входящих в такую пару функций тождественно равна 1; эти случаи уже исключены из рассмотрения. Отсюда следует, что для каждого фиксированного $r<2^{n-1}$ внутренняя сумма в (12), соответствующая случаю $k=2$, совпадает с

$$
2^{\left(\begin{array}{c}
n \\
2
\end{array}\right)+n} \sum_{\substack{q \in Q_{n} \\
q \neq 0}} N_{2^{n}}^{(2)}(w t(q), r)=S_{n}^{(2)}(r)
$$

где $w t(\cdot)$ означает вес функции, и

$$
N_{2^{n}}^{(2)}(w t(q), r)=\left|\left\{f \in \mathbb{F}_{2}^{V_{n}}: \max \{\rho(f, 0), \rho(f, q)\} \leqslant r\right\}\right| .
$$

Предложение 1. Пусть $b=\left(b_{1}, \ldots, b_{2^{n}}\right) \in \mathbb{F}_{2}^{V_{n}} u w t(b) \in\left\{1, \ldots, 2^{n}\right\}$, тогдa

- если $r<w t(b) / 2$, то $N_{2^{n}}^{(2)}(w t(b), r)=0$,

- если wt(b)/2 $\leqslant r<2^{n}$, то

$$
N_{2^{n}}^{(2)}(w t(b), r)=\sum_{m_{0}=0}^{r-w t(b) / 2} \sum_{m_{1}=\max \left\{w t(b)-\left(r-m_{0}\right), 0\right\}}^{\min \left\{r-m_{0}, w t(b)\right\}}\left(\begin{array}{c}
2^{n}-w t(b) \\
m_{0}
\end{array}\right)\left(\begin{array}{c}
w t(b) \\
m_{1}
\end{array}\right)
$$

(суммы, в которых верхний предел суммирования меньше нижнего, считаются равными нулю).

Доказательство. При фиксированной функции $b=\left(b_{1}, \ldots, b_{2^{n}}\right) \in \mathbb{F}_{2}^{V_{n}}$, сопоставим каждой функции $a=\left(a_{1}, \ldots, a_{2^{n}}\right) \in \mathbb{F}_{2}^{V_{n}}$ величины

$$
\begin{aligned}
& m_{0}=\left|\left\{i \in\left\{1, \ldots, 2^{n}\right\}: b_{i}=0, a_{i}=1\right\}\right|, \\
& m_{1}=\left|\left\{i \in\left\{1, \ldots, 2^{n}\right\}: b_{i}=1, a_{i}=1\right\}\right| .
\end{aligned}
$$

Число функций $a \in \mathbb{F}_{2}^{V_{n}}$ с фиксированными значениями $m_{0}$ и $m_{1}$ равно, очевидно, $\left(\begin{array}{c}2^{n}-w t(b) \\ m_{0}\end{array}\right)\left(\begin{array}{c}w t(b) \\ m_{1}\end{array}\right)$. Далее,

$$
\begin{aligned}
& \rho(a, 0)=m_{0}+m_{1}, \\
& \rho(a, b)=m_{0}+w t(b)-m_{1},
\end{aligned}
$$

то есть, при $w t(b) / 2 \leqslant r<n$ условия $\{\rho(a, 0) \leqslant r, \rho(a, b) \leqslant r\}$ эквивалентны условиям

$$
\left\{\begin{array} { l } 
{ 0 \leqslant m _ { 0 } \leqslant n - w t ( b ) , } \\
{ 0 \leqslant m _ { 1 } \leqslant w t ( b ) , } \\
{ m _ { 0 } + m _ { 1 } \leqslant r , } \\
{ m _ { 0 } + w t ( b ) - m _ { 1 } \leqslant r , }
\end{array} \Longleftrightarrow \left\{\begin{array}{l}
0 \leqslant m_{0} \leqslant r-w t(b) / 2, \\
0 \leqslant m_{1} \leqslant w t(b), \\
w t(b)-\left(r-m_{0}\right) \leqslant m_{1} \leqslant r-m_{0} .
\end{array}\right.\right.
$$

Значит,

$$
\begin{aligned}
N_{2^{n}}^{(2)}(w t(b), r) & =\left|\left\{a \in \mathbb{F}_{2}^{V_{n}}: \max \{\rho(a, 0), \rho(a, b)\} \leqslant r\right\}\right| \\
& =\sum_{m_{0}=0}^{r-w t(b) / 2} \sum_{m_{1}=\max \left\{w t(b)-\left(r-m_{0}\right), 0\right\}}^{\min \left\{r-m_{0}, w t(b)\right\}}\left(\begin{array}{c}
2^{n}-w t(b) \\
m_{0}
\end{array}\right)\left(\begin{array}{c}
w t(b) \\
m_{1}
\end{array}\right) .
\end{aligned}
$$


В силу (1) и предложения 1 , если $r<2^{n-3}$, то $N_{2^{n}}^{(2)}(w t(q), r)=0$ для любого $q \in \mathbb{Q}_{n}$, то есть $S_{n}^{(2)}(r)=0$ при $r<2^{n-3}=n_{1}^{-}$. Если $2^{n-3} \leqslant r<2^{n-1}$, то

$$
\begin{aligned}
& S_{n}^{(2)}(r)=2^{\left(\begin{array}{c}
n \\
2
\end{array}\right)+n}\left(\sum_{s=1}^{[n / 2]}\left(\left|W_{2 n_{s}^{-}}\right| N_{2^{n}}^{(2)}\left(2 n_{s}^{-}, r\right)+\left|W_{2 n_{s}^{+}}\right| N_{2^{n}}^{(2)}\left(2 n_{s}^{+}, r\right)\right)\right. \\
& \left.+\left|W_{2^{n-1}}\right| N_{2^{n}}^{(2)}\left(n_{0}^{+}, r\right)\right) \text {. }
\end{aligned}
$$

Рассмотрим последнее выражение при различных значениях $r$, принимая во внимание, что $N_{2^{n}}^{(2)}(k, r)=0$ при $r<k / 2$. При $n_{t}^{-} \leqslant r<n_{t+1}^{-}, t=1, \ldots,[n / 2]-1$, в (15) отличны от нуля лишь слагаемые, содержащие в аргументах $2 n_{s}^{-}, s=1, \ldots, t$, то есть, согласно принятым обозначениям,

$$
S_{n}^{(2)}(r)=2^{\left(\begin{array}{c}
n \\
2
\end{array}\right)+n} \sum_{s=1}^{t}\left|W_{2 n_{s}^{-}}\right| N_{2^{n}}^{(2)}\left(2 n_{s}^{-}, r\right)=A_{n}^{-}(t, r) .
$$

Если $n_{[n / 2]}^{-} \leqslant r<2^{n-2}$, то

$$
S_{n}^{(2)}(r)=2^{\left(\begin{array}{c}
n \\
2
\end{array}\right)+n} \sum_{s=1}^{[n / 2]}\left|W_{2 n_{s}^{-}}\right| N_{2^{n}}^{(2)}\left(2 n_{s}^{-}, r\right)=A_{n}^{-}([n / 2], r) .
$$

Если $r \geqslant 2^{n-2}$, то, в силу предложения 1 , поскольку $n_{0}^{+}=2^{n-1}$, последнее слагаемое в (15), а именно,

$$
2^{\left(\begin{array}{c}
n \\
2
\end{array}\right)+n}\left|W_{2^{n-1}}\right| N_{2^{n}}^{(2)}\left(n_{0}^{+}, r\right)=B_{n}(r),
$$

также отлично от нуля. При $n_{t}^{+} \leqslant r n_{t-1}^{+}, t=2, \ldots,[n / 2]$, в выражении (15) равными нулю оказываются слагаемые $\left|W_{2 n_{s}^{+}}\right| N_{2^{n}}^{(2)}\left(2 n_{s}^{+}, r\right), s=1, \ldots, t-1$, значит,

$$
\begin{aligned}
S_{n}^{(2)}(r) & =A_{n}^{-}([n / 2], r)+B_{n}(r)+\sum_{s=t}^{[n / 2]}\left|W_{2 n_{s}^{+}}\right| N_{2^{n}}^{(2)}\left(2 n_{s}^{+}, r\right) \\
& =A_{n}^{-}([n / 2], r)+B_{n}(r)+A_{n}^{+}(t, r) ;
\end{aligned}
$$

наконец, если $n_{1}^{+} \leqslant r<2^{n-1}$, то все слагаемые в (15) отличны от нуля.

Учитывая, что

$$
\left|W_{s}^{-}\right|=\left|W_{s}^{+}\right|, \quad\left|W_{2^{n-1}}\right|=2^{\left(\begin{array}{c}
n \\
2
\end{array}\right)+n+1}-\sum_{i \neq n_{0}^{+}}\left|W_{i}\right|,
$$

в силу (1), приходим к выражениям (9) и (11), что завершает доказательство теоремы 2.

Точные формулы для величин $N_{2^{n}}^{(1)}(r), S_{n}^{(2)}(r)$ в теоремах 1 и 2 даже при умеренных значениях $n$ слишком сложны для вычислений. Далее будут приведены простые оценки этих величин, позволяющие делать выводы о точности формул в теоремах 1 и 2 при $n, r \rightarrow \infty$. 
Для оценки $N_{2^{n}}^{(1)}(r)$ при $r \leqslant 2^{n-1}$ можно использовать неравенства из [1]:

$$
\begin{gathered}
\left(\begin{array}{c}
2^{n} \\
r
\end{array}\right)(1+q) \leqslant N_{2^{n}}^{(1)}(r) \leqslant\left(\begin{array}{c}
2^{n} \\
r
\end{array}\right) \frac{1}{1-q}, \quad q=\frac{r}{2^{n}-r+1}, \\
2^{2^{n}} \Phi\left(-\sqrt{2^{n} V\left(1-r / 2^{n-1}\right)}\right) \leqslant N_{2^{n}}^{(1)}(r) \leqslant 2^{2^{n}} \Phi\left(-\sqrt{2^{n} V\left(1-(r+1) / 2^{n-1}\right)}\right),
\end{gathered}
$$

И

$$
\begin{gathered}
\frac{2^{n 2^{n}}}{r^{r}\left(2^{n}-r\right)^{2^{n}-r} \sqrt{2^{n+1} \pi V\left(1-r / 2^{n-1}\right)}}\left(1-\frac{1}{2^{n} V\left(1-r / 2^{n-1}\right)}\right) \\
<N_{2^{n}}^{(1)}(r)<\frac{2^{n 2^{n}}}{\left.(r+1)^{r+1}\left(2^{n}-r-1\right)^{2^{n}-r-1} \sqrt{2^{n+1} \pi V\left(1-(r+1) / 2^{n-1}\right.}\right)},
\end{gathered}
$$

где

$$
\begin{aligned}
V(z) & =(1-z) \ln (1-z)+(1+z) \ln (1+z) \\
& =\sum_{k=1}^{\infty} \frac{z^{2 k}}{k(2 k-1)} \geqslant z^{2},
\end{aligned}
$$

и $\Phi(\cdot)$ - функция стандартного нормального распределения.

Нам потребуется также простая оценка для $N_{2^{n}}^{(2)}(k, r)$.

Предложение 2. Если $0 \leqslant k \leqslant 2^{n}, k$-четное число $u 0 \leqslant r \leqslant 2^{n-1}$, то

$$
N_{2^{n}}^{(2)}(k, r) \leqslant\left(\begin{array}{c}
k \\
k / 2
\end{array}\right)\left(\begin{array}{c}
2^{n}-k \\
r-k / 2
\end{array}\right) \frac{1+q_{k}}{\left(1-q_{k}\right)^{2}},
$$

гेe

$$
q_{k}=\frac{r-k / 2}{2^{n}-k / 2-r+1}<q=\frac{r}{2^{n}-r+1} .
$$

Доказательство. Чтобы получить верхнюю оценку для $N_{2^{n}}^{(2)}(k, r)$, заменим все слагаемые во внутренней сумме (14) их максимальным значением $\left(\begin{array}{c}k \\ k / 2\end{array}\right)$, положим

$$
q_{k}=\frac{\left(\begin{array}{c}
2^{n}-k \\
r-k / 2-1
\end{array}\right)}{\left(\begin{array}{c}
2^{n}-k \\
r-k / 2
\end{array}\right)}=\frac{r-k / 2}{2^{n}-k / 2-r+1}
$$

используем оценки

$$
\left(\begin{array}{c}
2^{n}-k \\
r-k / 2-m
\end{array}\right) \leqslant q_{k}^{m}\left(\begin{array}{c}
2^{n}-k \\
r-k / 2
\end{array}\right)
$$


и получим

$$
\begin{aligned}
N_{2^{n}}^{(2)}(k, r) & =\sum_{m_{0}=0}^{r-k / 2} \sum_{m_{1}=\max \left\{k-\left(r-m_{0}\right), 0\right\}}^{\min \left\{r-m_{0}, k\right\}}\left(\begin{array}{c}
2^{n}-k \\
m_{0}
\end{array}\right)\left(\begin{array}{c}
k \\
m_{1}
\end{array}\right) \\
& <\left(\begin{array}{c}
k \\
k / 2
\end{array}\right) \sum_{m=0}^{r-k / 2}\left(\begin{array}{c}
2^{n}-k \\
r-k / 2-m
\end{array}\right)(1+2 m) \\
& <\left(\begin{array}{c}
k \\
k / 2
\end{array}\right)\left(\begin{array}{c}
2^{n}-k \\
r-k / 2
\end{array}\right) \sum_{m=0}^{\infty} q_{k}^{m}(1+2 m) \\
& =\left(\begin{array}{c}
k \\
k / 2
\end{array}\right)\left(\begin{array}{c}
2^{n}-k \\
r-k / 2
\end{array}\right) \frac{1+q_{k}}{\left(1-q_{k}\right)^{2}} .
\end{aligned}
$$

Неравенство $q_{k}<q$ следует из того, что

$$
\begin{aligned}
q_{k} & =\frac{r-k / 2}{2^{n}-k / 2-r+1}=1-\frac{2^{n}-2 r+1}{2^{n}-k / 2-r+1} \\
& <1-\frac{2^{n}-2 r+1}{2^{n}-r+1}=\frac{r}{2^{n}-r+1}=q .
\end{aligned}
$$

Предложение 2 доказано.

Доказательство теоремы 1. Согласно теореме 2,

$$
2^{\left(\begin{array}{c}
n \\
2
\end{array}\right)+n+1} N_{2^{n}}^{(1)}(r)-S_{n}^{(2)}(r) \leqslant\left|\mathbb{F}_{2}\left(\mathbb{Q}_{n}, r\right)\right| \leqslant 2^{\left(\begin{array}{c}
n \\
2
\end{array}\right)+n+1} N_{2^{n}}^{(1)}(r),
$$

поэтому при

$$
Q(n, r)=\frac{S_{n}^{(2)}(r)}{2^{\left(\begin{array}{c}
n \\
2
\end{array}\right)+n+1} N_{2^{n}}^{(1)}(r)},
$$

получаем утверждение теоремы 1:

$$
2^{\left(\begin{array}{c}
n \\
2
\end{array}\right)+n+1} N_{2^{n}}^{(1)}(r)(1-Q(n, r)) \leqslant\left|\mathbb{F}_{2}\left(\mathbb{Q}_{n}, r\right)\right| \leqslant 2^{\left(\begin{array}{c}
n \\
2
\end{array}\right)+n+1} N_{2^{n}}^{(1)}(r) .
$$

Для завершения доказательства остается обосновать оценки величин $Q(n, r)$.

Из части (б) теоремы 2 следует, что

$$
Q(n, r)=0, \quad 0 \leqslant r<2^{n-3} .
$$

Теорема 3. Если

$$
n \geqslant 15, \quad r=2^{n-1}-c_{r} n \sqrt{2^{n-2} \ln 2} \geqslant 0, \quad c_{r}>1,
$$

mo

$$
Q(n, r)<\frac{2^{-n^{2}\left(c_{r}^{2}-3\right) / 6+n+1}}{n^{2}} \exp \left\{\frac{\left(c_{r} n\right)^{3}}{7 \cdot 2^{n / 2}}\right\}
$$


Доказательство. Учитывая (15) и то, что чем меньше расстояние между двумя функциями, тем больше функций попадает в пересечение их $r$-окрестностей, то есть при $r<2^{n-1}$, $s=1, \ldots,[n / 2]$

$$
N_{2^{n}}^{(2)}\left(2 n_{s}^{+}, r\right)<N_{2^{n}}^{(2)}\left(n_{0}^{+}, r\right)<N_{2^{n}}^{(2)}\left(2 n_{s}^{-}, r\right) \leqslant N_{2^{n}}^{(2)}\left(2 n_{1}^{-}, r\right),
$$

и поэтому

$$
\begin{aligned}
Q(n, r)= & \frac{1}{2 N_{2^{n}}^{(1)}(r)} \sum_{s=1}^{[n / 2]}\left(\left|W_{2 n_{s}^{-}}\right| N_{2^{n}}^{(2)}\left(2 n_{s}^{-}, r\right)+\left|W_{2 n_{s}^{+}}\right| N_{2^{n}}^{(2)}\left(2 n_{s}^{+}, r\right)\right) \\
& +\frac{1}{2 N_{2^{n}}^{(1)}(r)}\left|W_{2^{n-1}}\right| N_{2^{n}}^{(2)}\left(n_{0}^{+}, r\right) \\
< & \frac{1}{2 N_{2^{n}}^{(1)}(r)} \sum_{s=1}^{[n / 2]}\left(\left|W_{2 n_{s}^{-}}\right| N_{2^{n}}^{(2)}\left(2 n_{1}^{-}, r\right)+\left|W_{2 n_{s}^{+}}\right| N_{2^{n}}^{(2)}\left(2 n_{1}^{-}, r\right)\right) \\
& +\frac{1}{2 N_{2^{n}}^{(1)}(r)}\left|W_{2^{n-1}}\right| N_{2^{n}}^{(2)}\left(2 n_{1}^{-}, r\right) .
\end{aligned}
$$

Принимая во внимание равенства

$$
\left|W_{2 n_{s}^{-}}\right|=\left|W_{2 n_{s}^{+}}\right|, \quad\left|W_{2^{n-1}}\right|=2^{\left(\begin{array}{l}
n \\
2
\end{array}\right)+n+1}-\sum_{i \neq n_{0}^{+}}\left|W_{i}\right|
$$

а также оценки величин $N_{2^{n}}^{(1)}(r)$ и $N_{2^{n}}^{(2)}\left(2 n_{1}^{-}, r\right)$, получим, что

$$
\begin{aligned}
Q(n, r) & <\frac{2^{\left(\begin{array}{c}
n \\
2
\end{array}\right)+n} N_{2^{n}}^{(2)}\left(2 n_{1}^{-}, r\right)}{N_{2^{n}}^{(1)}(r)} \\
& \leqslant \frac{2^{\left(\begin{array}{c}
n \\
2
\end{array}\right)+n}\left(\begin{array}{c}
2 n_{1}^{-} \\
n_{1}^{-}
\end{array}\right)\left(\begin{array}{c}
2^{n}-2 n_{1}^{-} \\
r-n_{1}^{-}
\end{array}\right)\left(1+q_{2 n_{1}^{-}}\right) /\left(1-q_{2 n_{1}^{-}}\right)^{2}}{\left(\begin{array}{c}
2^{n} \\
r
\end{array}\right)(1+q)},
\end{aligned}
$$

где

$$
q_{2 n_{1}^{-}}=\frac{r-n_{1}^{-}}{2^{n}-n_{1}^{-}-r+1}<q=\frac{r}{2^{n}-r+1} .
$$

Так как

$$
\begin{aligned}
\left(\begin{array}{c}
2^{n} \\
r
\end{array}\right) & =\left(\begin{array}{c}
2^{n} \\
2^{n-1}
\end{array}\right) \prod_{k=1}^{2^{n-1}-r} \frac{r+k}{2^{n-1}+k}, \\
\left(\begin{array}{c}
2^{n}-2 m \\
r-m
\end{array}\right) & =\left(\begin{array}{c}
2^{n}-2 m \\
2^{n-1}-m
\end{array}\right) \prod_{k=1}^{2^{n-1}-r} \frac{r-m+k}{2^{n-1}-m+k},
\end{aligned}
$$

справедливо равенство

$$
\frac{\left(\begin{array}{c}
2 m \\
m
\end{array}\right)\left(\begin{array}{c}
2^{n}-2 m \\
r-m
\end{array}\right)}{\left(\begin{array}{c}
2^{n} \\
r
\end{array}\right)}=\frac{\left(\begin{array}{c}
2 m \\
m
\end{array}\right)\left(\begin{array}{c}
2^{n}-2 m \\
2^{n-1}-m
\end{array}\right)}{\left(\begin{array}{c}
2^{n} \\
2^{n-1}
\end{array}\right)} \prod_{k=1}^{2^{n-1}-r} \frac{(r-m+k)\left(2^{n-1}+k\right)}{(r+k)\left(2^{n-1}-m+k\right)} .
$$


С помощью уточненной формулы Стирлинга (см. [5], гл. 2, §9)

$$
\sqrt{2 \pi m}\left(\frac{m}{e}\right)^{m} e^{1 /(12 m+1)}<m ! \sqrt{2 \pi m}\left(\frac{m}{e}\right)^{m} e^{1 / 12 m},
$$

оценим первую дробь в правой части (21):

$$
\begin{aligned}
\frac{\left(\begin{array}{c}
2 m \\
m
\end{array}\right)\left(\begin{array}{c}
2^{n}-2 m \\
2^{n-1}-m
\end{array}\right)}{\left(\begin{array}{c}
2^{n} \\
2^{n-1}
\end{array}\right)} & =\frac{(2 m) !\left(2^{n}-2 m\right) !}{2^{n} !}\left(\frac{2^{n-1} !}{m !\left(2^{n-1}-m\right) !}\right)^{2} \\
& <\frac{1}{\sqrt{\pi m\left(1-2 m / 2^{n}\right)}} \exp \left\{\frac{1}{24 m\left(1-2 m / 2^{n}\right)}+\frac{1}{3 \cdot 2^{n}}\right\} .
\end{aligned}
$$

Значит, при $s \geqslant 1$ и $m=n_{s}^{-}$или $m=n_{s}^{+}$

$$
\begin{aligned}
\frac{\left(\begin{array}{c}
2 m \\
m
\end{array}\right)\left(\begin{array}{c}
2^{n}-2 m \\
2^{n-1}-m
\end{array}\right)}{\left(\begin{array}{c}
2^{n} \\
2^{n-1}
\end{array}\right)} & <\frac{1}{\sqrt{2^{n-3} \pi\left(1-1 / 2^{2 s}\right)}} \exp \left\{\frac{1}{24 \cdot 2^{n-3}\left(1-1 / 2^{2 s}\right)}+\frac{1}{3 \cdot 2^{n}}\right\} \\
& \leqslant \frac{e^{7 /\left(9 \cdot 2^{n}\right)}}{\sqrt{\pi 2^{n-3} 3 / 4}} .
\end{aligned}
$$

Нетрудно проверить, что при $y=2^{n-1}-r$

$$
\frac{\left(2^{n-1}+k\right)(r-m+k)}{(r+k)\left(2^{n-1}-m+k\right)}=1-\frac{m y}{\left(2^{n-1}+k-m\right)\left(2^{n-1}+k-y\right)} .
$$

Таким образом, сомножители произведения (21) строго возрастают по $k$. Поэтому

$$
\prod_{k=1}^{2^{n-1}-r} \frac{(r-m+k)\left(2^{n-1}+k\right)}{(r+k)\left(2^{n-1}-m+k\right)} \leqslant\left(\frac{2^{n}-r}{2^{n-1}} \frac{2^{n-1}-m}{2^{n}-r-m}\right)^{2^{n-1}-r},
$$

что убывает при растущем $m$, так как $r<2^{n-1}$.

Из (20), (22) и того, что $q_{k}<q$ и

$$
1-q_{k}=\frac{2^{n}-2 r+1}{2^{n}-k / 2-r+1} \geqslant \frac{2^{n}-2 r}{2^{n}-k / 2-r}, \quad r \geqslant k / 2,
$$

следует оценка

$$
Q(n, r)<\frac{2^{\left(\begin{array}{c}
n \\
2
\end{array}\right)+n+1} e^{7 /\left(9 \cdot 2^{n}\right)}}{\sqrt{3 \pi 2^{n-3}}}\left(\frac{2^{n}-r}{2^{n-1}} \frac{2^{n-1}-n_{1}^{-}}{2^{n}-r-n_{1}^{-}}\right)^{2^{n-1}-r}\left(\frac{2^{n}-n_{1}^{-}-r}{2^{n}-2 r}\right)^{2} .
$$

Оценим сомножители в (24), положив $y=2^{n-1}-r$. По условию

$$
y=c_{r} n \sqrt{2^{n-2} \ln 2},
$$

тогда при $n \geqslant 15$ и $c_{r}>1$

$$
\left(\frac{2^{n}-n_{1}^{-}-r}{2^{n-1}-r}\right)^{2}=\left(\frac{3 \cdot 2^{n-3}}{y}+1\right)^{2}<\left(\frac{3 \cdot 2^{n / 2}}{4 n \sqrt{\ln 2}}+1\right)^{2}<\frac{2^{n}}{n^{2}} .
$$


Используя неравенства

$$
1-x \leqslant e^{-x}, \quad 8 \sqrt{\ln 2}<7, \quad \frac{7 \ln 2}{36}<\frac{1}{7},
$$

получим, что

$$
\begin{aligned}
\left(\frac{2^{n}-r}{2^{n-1}} \frac{2^{n-1}-n_{1}^{-}}{2^{n}-r-n_{1}^{-}}\right)^{2^{n-1}-r} & =\left(\frac{3}{4}\right)^{y}\left(\frac{2^{n-1}+y}{3 \cdot 2^{n-3}+y}\right)^{y}=\left(1-\frac{y}{12 \cdot 2^{n-3}+4 y}\right)^{y} \\
& <\exp \left\{-\frac{y^{2}}{3 \cdot 2^{n-1}+4 y}\right\}<\exp \left\{-\frac{c_{r}^{2} n^{2}(\ln 2) / 6}{1+(7 / 6) c_{r} n \sqrt{2^{-n}}}\right\} \\
& =2^{-c_{r}^{2} n^{2} / 6} \exp \left\{\frac{(7 / 6) c_{r}^{3} n^{3}((\ln 2) / 6) \sqrt{2^{-n}}}{1+(7 / 6) c_{r} n \sqrt{2^{-n}}}\right\} \\
& <2^{-c_{r}^{2} n^{2} / 6} \exp \left\{\frac{\left(c_{r} n\right)^{3}}{7 \cdot 2^{n / 2}}\right\} .
\end{aligned}
$$

Значит, при $n \geqslant 15$ и

$$
r=2^{n-1}-c_{r} n \sqrt{2^{n-2} \ln 2} \geqslant 0, \quad c_{r}>1,
$$

согласно (25), (26),

$$
Q(n, r)<\frac{2^{\left(\begin{array}{c}
n \\
2
\end{array}\right)+n+1} e^{7 /\left(9 \cdot 2^{n}\right)}}{\sqrt{3 \pi 2^{n-3}}} 2^{-c_{r}^{2} n^{2} / 6} \exp \left\{\frac{\left(c_{r} n\right)^{3}}{7 \cdot 2^{n / 2}}\right\} \frac{2^{n}}{n^{2}} .
$$

Учитывая, что при $n \geqslant 15$

$$
\frac{e^{7 /\left(9 \cdot 2^{n}\right)}}{\sqrt{3 \pi / 2}}<\frac{1}{2}
$$

упростим оценку сверху для $Q(n, r)$ :

$$
Q(n, r)<\frac{2^{-n^{2}\left(c_{r}^{2}-3\right) / 6+n+1}}{n^{2}} \exp \left\{\frac{\left(c_{r} n\right)^{3}}{7 \cdot 2^{n / 2}}\right\} .
$$

Теорема 3 доказана.

Доказательство следствия 1. Согласно определению (8),

$$
x(n, r)=\frac{1}{2}\left(\frac{1}{2^{n-2}}\left(2^{n-1}-r\right)^{2}-\left(n^{2}+n\right) \ln 2+\ln \left(\frac{n^{2} \pi \ln 2}{\sqrt[4]{2}}\right)\right) .
$$

Поэтому при $n \rightarrow \infty$

$$
e^{-x(n, r)}=\frac{2^{\left(n^{2}+n\right) / 2+1 / 8}}{n \sqrt{\pi \ln 2}} e^{-\left(2^{n-1}-r\right)^{2} / 2^{n-1}} \rightarrow 0,
$$

если

$$
\frac{\left(2^{n-1}-r\right)^{2}}{2^{n-1}}-\frac{n^{2}+n}{2} \ln 2 \rightarrow \infty
$$


а последнее условие выполняется, если

$$
n \rightarrow \infty, \quad \exists c>1: \quad 0 \leqslant r<2^{n-1}-c \sqrt{2^{n-2}\left(n^{2}+n\right) \ln 2} .
$$

Значит, при условиях (27) и достаточно больших $n$

$$
1-e^{-e^{-x(n, r)}}>e^{-x(n, r)} \frac{1}{\sqrt[8]{2}}=\frac{2^{\left(n^{2}+n\right) / 2}}{n \sqrt{\pi \ln 2}} e^{-\left(2^{n-1}-r\right)^{2} / 2^{n-1}} .
$$

С другой стороны, из неравенства в доказательстве следствия 1 в [1] следует оценка

$$
N_{2^{n}}^{(1)}(r)<\frac{2^{2^{n}+n / 2-3 / 2}}{\sqrt{\pi}\left(2^{n-1}-r-1\right)} e^{-\left(2^{n-1}-r-1\right)^{2} / 2^{n-1}} .
$$

Так как

$$
\left|\mathbb{F}_{2}\left(\mathbb{Q}_{n}, r\right)\right|<2^{\left(\begin{array}{c}
n \\
2
\end{array}\right)+n+1} N_{2^{n}}^{(1)}(r),
$$

из (28) и последнего неравенства следует, что при условиях (27) и достаточно больших $n$

$$
\begin{aligned}
\frac{\left|\mathbb{F}_{2}\left(\mathbb{Q}_{n}, r\right)\right|}{2^{2^{n}}\left(1-e^{\left.-e^{-x(n, r)}\right)}\right.} & <\frac{2^{2^{n}+3 n / 2+\left(\begin{array}{c}
n \\
2
\end{array}\right)}\left(\sqrt{2 \pi}\left(2^{n-1}-r-1\right)\right)^{-1} e^{-\left(2^{n-1}-r-1\right)^{2} / 2^{n-1}}}{\left(2^{2^{n}+\left(n^{2}+n\right) / 2} /(n \sqrt{\pi \ln 2})\right) e^{-\left(2^{n-1}-r\right)^{2} / 2^{n-1}}} \\
& <\frac{2^{(n-1) / 2} n \sqrt{\ln 2}}{2^{n-1}-r-1} e^{\left(2^{n}-2 r\right) / 2^{n-1}} \\
& <\frac{2^{(n-1) / 2} n \sqrt{\ln 2}}{c \sqrt{2^{n-2}\left(n^{2}+n\right) \ln 2}-1} e^{\left(2^{n}-2 r\right) / 2^{n-1}}<\frac{1,5}{c} .
\end{aligned}
$$

Следствие 1 доказано.

Для $Q(n, r)$ при $r<2^{n-2}$ справедливы и более точные оценки.

Теорема 4. Если $n \geqslant 3, n_{t}^{-} \leqslant r<n_{t+1}^{-}, t=1, \ldots,[n / 2]-1$, тогда

$$
Q(n, r)<\frac{18,75}{\sqrt{3 \pi 2^{n+3}}} \exp \left\{-\frac{2^{n-3}\left(1+1 / 2^{t+1}\right)^{2}}{5+1 / 2^{t-1}}+t(2 n-2 t+1) \ln 2+\ln t+\frac{7}{9 \cdot 2^{n}}\right\} .
$$

Если $n_{[n / 2]}^{-} \leqslant r<2^{n-2}$, тогда

$$
Q(n, r)<\frac{6,25}{\sqrt{3 \pi 2^{n+1}}} \exp \left\{-\frac{2^{n-3}}{5+1 / 2^{[n / 2]-1}}+2\left[\frac{n}{2}\right]\left(n-\left[\frac{n}{2}\right]+\frac{1}{2}\right) \ln 2+\ln \left[\frac{n}{2}\right]+\frac{7}{9 \cdot 2^{n}}\right\} .
$$

Доказательство. Согласно оценкам (16), (17) и определению (18),

$$
\begin{aligned}
Q(n, r) \leqslant \sum_{s=1}^{[n / 2]} & \frac{\left|W_{2 n_{s}^{-}}\right|}{2\left(\begin{array}{c}
2^{n} \\
r
\end{array}\right)(1+q)}\left(\left(\begin{array}{c}
2 n_{s}^{-} \\
n_{s}^{-}
\end{array}\right)\left(\begin{array}{c}
2^{n}-2 n_{s}^{-} \\
r-n_{s}^{-}
\end{array}\right) \frac{1+q_{2 n_{s}^{-}}}{\left(1-q_{2 n_{s}^{-}}\right)^{2}}\right. \\
& \left.+\left(\begin{array}{c}
2 n_{s}^{+} \\
n_{s}^{+}
\end{array}\right)\left(\begin{array}{c}
2^{n}-2 n_{s}^{+} \\
r-n_{s}^{+}
\end{array}\right) \frac{1+q_{2 n_{s}^{+}}}{\left(1-q_{2 n_{s}^{+}}\right)^{2}}\right) \\
& +\left|W_{n_{0}^{+}}\right| \frac{\left(\begin{array}{c}
n_{0}^{+} \\
n_{0}^{+} / 2
\end{array}\right)\left(\begin{array}{c}
n_{0}^{+} \\
r-n_{0}^{+} / 2
\end{array}\right)}{2\left(\begin{array}{c}
2^{n} \\
r
\end{array}\right)(1+q)} \frac{1+q_{n_{0}^{+}}}{\left(1-q_{n_{0}^{+}}\right)^{2}} .
\end{aligned}
$$


Так как

$$
q_{k}=\frac{r-k / 2}{2^{n}-k / 2-r+1}<q=\frac{r}{2^{n}-r+1}
$$

и

$$
1-q_{k}=\frac{2^{n}-2 r+1}{2^{n}-k / 2-r+1} \geqslant \frac{2^{n}-2 r}{2^{n}-k / 2-r}, \quad r \geqslant k / 2,
$$

получаем, что

$$
\begin{gathered}
Q(n, r)<\sum_{s=1}^{[n / 2]} \frac{\left|W_{2 n_{s}^{-}}\right|}{2\left(\begin{array}{c}
2^{n} \\
r
\end{array}\right)}\left(\left(\begin{array}{c}
2 n_{s}^{-} \\
n_{s}^{-}
\end{array}\right)\left(\begin{array}{c}
2^{n}-2 n_{s}^{-} \\
r-n_{s}^{-}
\end{array}\right)\left(\frac{2^{n}-n_{s}^{-}-r}{2^{n}-2 r}\right)^{2}\right. \\
\left.+\left(\begin{array}{c}
2 n_{s}^{+} \\
n_{s}^{+}
\end{array}\right)\left(\begin{array}{c}
2^{n}-2 n_{s}^{+} \\
r-n_{s}^{+}
\end{array}\right)\left(\frac{2^{n}-n_{s}^{+}-r}{2^{n}-2 r}\right)^{2}\right) \\
+\left|W_{n_{0}^{+}}\right| \frac{\left(\begin{array}{c}
n_{0}^{+} \\
n_{0}^{+} / 2
\end{array}\right)\left(\begin{array}{c}
n_{0}^{+} \\
r-n_{0}^{+} / 2
\end{array}\right)}{2\left(\begin{array}{c}
2^{n} \\
r
\end{array}\right)}\left(\frac{2^{n}-n_{0}^{+} / 2-r}{2^{n}-2 r}\right)^{2} .
\end{gathered}
$$

Для оценки последнего слагаемого в (29) используем формулу из доказательства теоремы 5 на с. 17 в [1], откуда следует, что при $n \geqslant 8$

$$
\frac{\left(\begin{array}{c}
n_{0}^{+} \\
n_{0}^{+} / 2
\end{array}\right)\left(\begin{array}{c}
2^{n}-n_{0}^{+} \\
2^{n-1}-n_{0}^{+} / 2
\end{array}\right)}{\left(\begin{array}{c}
2^{n} \\
2^{n-1}
\end{array}\right)}=\frac{\left(\begin{array}{c}
2^{n-1} \\
2^{n-2}
\end{array}\right)}{\left(\begin{array}{c}
2^{n} \\
2^{n-1}
\end{array}\right)}<\frac{8}{5 \cdot 2^{n / 2}} .
$$

Объединение оценок (22), (23), полученных в доказательстве теоремы 3, приводит к неравенству

$$
\begin{aligned}
Q(n, r)<\sum_{s=1}^{[n / 2]} & \frac{\left|W_{2 n_{s}^{-}}\right|}{2} \frac{e^{7 /\left(9 \cdot 2^{n}\right)}}{\sqrt{\pi 2^{n-3} 3 / 4}}\left(\left(\frac{2^{n}-r}{2^{n-1}} \frac{2^{n-1}-n_{s}^{-}}{2^{n}-r-n_{s}^{-}}\right)^{y}\left(\frac{2^{n}-n_{s}^{-}-r}{2^{n}-2 r}\right)^{2}\right. \\
& \left.+\left(\frac{2^{n}-r}{2^{n-1}} \frac{2^{n-1}-n_{s}^{+}}{2^{n}-r-n_{s}^{+}}\right)^{y}\left(\frac{2^{n}-n_{s}^{+}-r}{2^{n}-2 r}\right)^{2}\right) \\
& +\frac{\mid W_{n_{0}^{+}}}{2} \frac{8}{5 \cdot 2^{n / 2}}\left(\frac{2^{n}-r}{2^{n-1}} \frac{2^{n-1}-n_{0}^{+} / 2}{2^{n}-r-n_{0}^{+} / 2}\right)^{y}\left(\frac{2^{n}-n_{0}^{+} / 2-r}{2^{n}-2 r}\right)^{2} .
\end{aligned}
$$

Обозначив $y=2^{n-1}-r$, из неравенства выше получим

$$
\begin{aligned}
Q(n, r)< & \frac{e^{7 /\left(9 \cdot 2^{n}\right)}}{\sqrt{\pi 2^{n-3} 3 / 4}} \sum_{s=1}^{[n / 2]} \frac{1}{8}\left|W_{2 n_{s}^{-}}\right|\left(\left(\frac{\left(2^{n-1}-n_{s}^{-}\right)\left(2^{n-1}+y\right)}{2^{n-1}\left(2^{n-1}+y-n_{s}^{-}\right)}\right)^{y}\left(\frac{2^{n-1}-n_{s}^{-}+y}{y}\right)^{2}\right. \\
& \left.+\left(\frac{\left(2^{n-1}-n_{s}^{+}\right)\left(2^{n-1}+y\right)}{2^{n-1}\left(2^{n-1}+y-n_{s}^{+}\right)}\right)^{y}\left(\frac{2^{n-1}-n_{s}^{+}+y}{y}\right)^{2}\right) \\
& +\frac{\left|W_{n_{0}^{+}}\right|}{5 \cdot 2^{n / 2}}\left(1-\frac{y}{2^{n-1}+2 y}\right)^{y}\left(\frac{2^{n-2}}{y}+1\right)^{2}
\end{aligned}
$$


Так как

$$
N_{2^{n}}^{(2)}\left(2 n_{s}^{+}, r\right)=0, \quad N_{2^{n}}^{(2)}\left(n_{0}^{+}, r\right)=0,
$$

при $2^{n-1}-n_{t+1}^{-}<y \leqslant 2^{n-1}-n_{t}^{-}$, то все слагаемые в (19) равны нулю, кроме слагаемых, содержащих $n_{s}^{-}, s=1, \ldots, t$, значит при $n_{t}^{-} \leqslant r<n_{t+1}^{-}$

$$
Q(n, r)<\frac{e^{7 /\left(9 \cdot 2^{n}\right)}}{\sqrt{\pi 2^{n-3} 3 / 4}} \sum_{s=1}^{t} \frac{1}{8}\left|W_{2 n_{s}^{-}}\right|\left(\frac{\left(2^{n-1}-n_{s}^{-}\right)\left(2^{n-1}+y\right)}{2^{n-1}\left(2^{n-1}+y-n_{s}^{-}\right)}\right)^{y}\left(\frac{2^{n-1}-n_{s}^{-}}{y}+1\right)^{2} .
$$

Далее оценим сверху по порядку сомножители в (30), начиная с последнего, при $s, t \geqslant 1$ и $2^{n-1}-n_{t+1}^{-}<y \leqslant 2^{n-1}-n_{t}^{-}$:

$$
\left(\frac{2^{n-1}-n_{s}^{-}}{y}+1\right)^{2}<\left(\frac{2^{n-1}-n_{1}^{-}}{2^{n-1}-n_{t+1}^{-}}+1\right)^{2}=\left(\frac{3 / 2}{1+1 / 2^{t+1}}+1\right)^{2}<6,25
$$

так как $n_{1}^{-}<n_{s}^{-}$и

$$
1-x \leqslant e^{-x}
$$

получаем, что

$$
\begin{aligned}
\left(\frac{\left(2^{n-1}-n_{s}^{-}\right)\left(2^{n-1}+y\right)}{2^{n-1}\left(2^{n-1}+y-n_{s}^{-}\right)}\right)^{y} & \leqslant\left(\frac{\left(2^{n-1}-n_{1}^{-}\right)\left(2^{n-1}+y\right)}{2^{n-1}\left(2^{n-1}+y-n_{1}^{-}\right)}\right)^{y} \\
& =\left(1-\frac{n_{s}^{-}}{2^{n-1}}\right)^{y}\left(1+\frac{n_{s}^{-}}{2^{n-1}+y-n_{s}^{-}}\right)^{y} \\
& =\left(\frac{3}{4}\right)^{y}\left(\frac{4 \cdot 2^{n-3}+y}{3 \cdot 2^{n-3}+y}\right)^{y} \\
& =\left(1-\frac{y}{12 \cdot 2^{n-3}+4 y}\right)^{y} \\
& \leqslant \exp \left\{-\frac{y^{2}}{3 \cdot 2^{n-1}+4 y}\right\}
\end{aligned}
$$

оценку $\left|W_{2 n_{s}^{-}}\right|$получим из замечания 1.

При $n_{t}^{-} \leqslant r<n_{t+1}^{-}, t=1, \ldots,[n / 2]-1$, объединяя оценки сомножителей в (30), учитывая, что

$$
\left|W_{2 n_{s}^{-}}\right| \leqslant\left|W_{t}^{-}\right|, \quad 1 \leqslant s \leqslant t
$$

и

$$
2^{n-1}-n_{t}^{-} \geqslant y=2^{n-1}-r>2^{n-1}-n_{t+1}^{-},
$$

получаем, что

$$
\begin{aligned}
Q(n, r) & <\frac{6,25 e^{7 /\left(9 \cdot 2^{n}\right)}}{\sqrt{3 \pi 2^{n-3} / 4}} \frac{3 t \cdot 2^{2 n t-2 t^{2}+t-1}}{8} \exp \left\{-\frac{y^{2}}{3 \cdot 2^{n-1}+4 y}\right\} \\
& <\frac{18,75}{\sqrt{3 \pi 2^{n+3}}} \exp \left\{-\frac{2^{n-2}\left(1+1 / 2^{t+1}\right)^{2}}{10+4 / 2^{t}}+t(2 n-2 t+1) \ln 2+\ln t+\frac{7}{9 \cdot 2^{n}}\right\} .
\end{aligned}
$$


При $n_{[n / 2]}^{-} \leqslant r<2^{n-2}$, пользуясь второй оценкой из замечания 1 ,получаем, что

$$
\begin{aligned}
Q(n, r) & <\frac{6,25 e^{7 /\left(9 \cdot 2^{n}\right)}}{\sqrt{3 \pi 2^{n-3} / 4}} \frac{[n / 2] 2^{2 n[n / 2]-2[n / 2]^{2}+[n / 2]}}{8} \exp \left\{-\frac{y^{2}}{3 \cdot 2^{n-1}+4 y}\right\} \\
& <\frac{6,25}{\sqrt{3 \pi 2^{n+1}}} \exp \left\{-\frac{2^{n-2}}{10+4 / 2^{[n / 2]}}+2\left[\frac{n}{2}\right]\left(n-\left[\frac{n}{2}\right]+\frac{1}{2}\right) \ln 2+\ln \left[\frac{n}{2}\right]+\frac{7}{9 \cdot 2^{n}}\right\} .
\end{aligned}
$$

Теорема 4 доказана.

Автор признателен А. М. Зубкову за постановку задачи и внимание.

\section{Список литературы}

1. Зубков А. М., Серов А. А., Оценки числа булевых функций, имеющих аффинные приближения заданной точности. Дискретная математика (2010) 22, №5, 3-19.

2. Логачев О. А., Сальников А. А., Ященко В. В., Булевы функици в теории кодирования и криптологии. МЦНМО, Москва, 2004.

3. Мак-Вильямс Ф. Дж., Слоэн Н. Дж. А., Теория кодов, исправляющих ошибки. Связь, Москва, 1979.

4. Рязанов Б. В., Чечета С. И., О приближении случайной булевой функции множеством квадратичных форм. Дискретная математика (1995) 7, №3, 129-145.

5. Феллер В., Введение в теорию вероятностей и ее приложения. Мир, Москва, 1984.

Статья поступила 19.12.2011. 